\title{
Behavior to Dynamic Loads of Multi-layer Composite Structures
}

\author{
VICTOR GEANTA ${ }^{1}$, IONELIA VOICULESCU ${ }^{2 *}$, TUDOR CHERECHES ${ }^{3}$, TEODORA ZECHERU ${ }^{4}$, LIVIU MATACHE ${ }^{5}$, ADRIAN ROTARIU ${ }^{5}$ \\ 'University Politehnica of Bucharest, Faculty of Materials Science and Engineering, 313 Splaiul Independentei, 060042, Romania \\ 2University Politehnica of Bucharest, Faculty of Engineering and Management of Technological Systems, 313 Splaiul Independentei \\ 060042, Bucharest, Romania \\ 3UPS PILOT ARM, Romania, 2 Laminorului Str., 130089, Targoviste, Romania \\ ${ }^{4}$ Scientific Research Center for CRBN Defence and Ecology Bucharest, 225 Oltenitei Road, 041309, Bucharest, Romania \\ ${ }_{5}^{5}$ Military Technical Academy Ferdinand I, 39-49 George Cosbuc Blvd., 050141, Bucharest, Romania
}

\begin{abstract}
The explosive effect and high velocity penetration of the ballistic projectiles of various sizes, design and compositions, on impact with different targets (armors composed of a combination of different metals) are complex. Both practical experiments and mathematical modeling of the phenomena associated to the interaction projectile-target are required to estimate their effect or to design more efficient projectiles and armor. In this study, the basic element of the simulation model is an incendiary projectile of caliber $7.62 \mathrm{~mm}$ with medium piercing power, launched with a maximum speed of $750 \mathrm{~ms}^{-1}$ on the multi-material target, which contains 4 different layers assembled into a ballistic cassette made of aluminum. The purpose of this ballistic cassette is to ensure a better contact and handling of multi-layer materials. The proposed model was calculated using mathematical modeling and empirical material constants to describe the nonlinear transitory impact process. Mathematical simulation of the impact between the projectile and target during impact shows that the projectile moves sequentially through the ballistic package, causing perforation, plastic deformation and heating, the resulting fragments being then expelled into the space around the target. The model indicates that the projectile will penetrate the front aluminum plate, as well as the AlCrFeCoNi and steel plates, but will be stopped by the aluminum backing plate. The real impact tests carried out using the ballistic cassette at dynamic impact with the $7.62 \mathrm{~mm}$ incendiary projectile confirm the model assumptions, which prove the capacity of the composite model to safely stop the projectile.
\end{abstract}

Keywords: modeling, dynamic impact, composed structures, high entropy alloy, ballistic cassette

The simulation and modeling methods allow the qualitative and quantitative study of the most complex mechanical, physical and chemical processes and phenomena. Through them, the system dynamic development and behavior could be estimated. In the area of materials science, the simulations allow the researcher to determine the outcome of material - system interaction in dynamic conditions, such as the impactrelation between projectile and target.

Split Hopkinson Pressure Bar (SHPB) is the most widely used method to describe the results of different materials samples exposed to medium and high speed shaping [1 -6]. The best described SHPB process induces unidirectional pressure in the target sample by the simultaneous opposing impact of two bars. The impact generates an elastic wave in the impacting bar which is partially transferred to the sample and partially reflected by the transition bar. Sensors installed at the ends of the bars measure the generated energy, and the results will allow the shaping of the energetic phenomena and the estimation of the generated forces. The SHPB method has data accuracy shortfalls related to noise level, characteristic wave length dispersion and a number of other specific characteristics [7 - 9].

The professional publications have dedicated extensive attention to the phenomena and processes arising during armor piercing projectile impact with materials of various compositions. Such research is focused on various types of high impact perforator projectiles and projectiles fragments, as well as the behavior vs. various impact targets such as armed concrete, metal plates and composite structures. The mathematical simulation using a limited number of preset characteristics, offers the possibility to study the impact and deformation process in real time and estimate the area of target crack by analyzing the depth of the penetration, calculate the residual speed of the projectile or fragments, calculating the deceleration profile as function of the initial launching speed. The characteristics of the impact area, cracks, craters and adjacent zones, are in general good agreement with the simulation data, leading to a good description of the complex interaction between the projectile and target [10]

An alternative to dynamic or static simulations is provided by compression tests. Static or dynamic compression tests permit the design and use of SHPB to study the deformation gradient at various values of temperature. Using lanthanum cylindrical specimens and employing the Johnson-Cook (J-K) equations, the specific deformation characteristics could be easily calculated. J-K compression equations calculated in the SHPB tests for lanthanum sample were calibrated through numeric simulations and the results confirmed large deformations when exposed to complex pressure tests. Based on the static and dynamic testresults (MTS) using the pulling test on a divided Hopkinson lanthanum sample, the tractor J-K equations were calculated. The reflected and transferred wave of the PSHB tests resulted from the numeric simulation for lanthanum sample, using the speed of deformation as function of crack failure and was confirmed by the subsequentexperiments. The relationship between the dynamic crack failure and the speed of tractor force was pointed out as critical. SEM analysis of the fractured surface showed the crack failure mechanism becomes erratical with increased speed of the applied effort [11, 23 - 24].

\footnotetext{
*email: ioneliav@yahoo.co.uk
} 
One objective of the other study was modeling the mass loss of the projectile nose when the projectile hits a defined target at high velocity. Using a semi-empiric model determined that the mass loss percentage is linear, depending on the projectile speed, and the depth of the penetration is directly dependent on the projectile nose mass loss [12].

Quantitative evaluation of physical, chemical, mechanical, etc., phenomena and processes can be successfully done by numeric simulation and mathematical modeling. In this study, the basic element of the simulation model is an incendiary projectile of caliber $7.62 \mathrm{~mm}$ with medium armor piercing power, launched with a maximum speed of $750 \mathrm{~ms}^{-1}$ on the multi-material target which contains 4 different layers (Al + AlCrFeCoNi + Armored Steel + Al) assembled into a ballistic cassette made of aluminum. The model and experiments indicate that the projectile can penetrate the front aluminum plate, as well as the AlCrFeCoNi and steel plates, but is stopped by the last back aluminum plate.

\section{Experimental part}

\section{Discrete mathematics used to study the physical} simulation model

To simulate the system interaction in terms of mechanics of continuous medium and to increase the chances of a representative simulation model, the basic assumptions for the target are the physical properties, encompassed in the equations describing the properties of the chosen materials and the proprieties of standard used projectile produced for tactical weapons (fig. 1). Discrete mathematics offers the opportunity to study and describe the system using geometry and algebra equations to transform the continuous aspect of the interaction projectile - BT (Ballistic Target) described by integer and differential equations into discrete, isolated events.

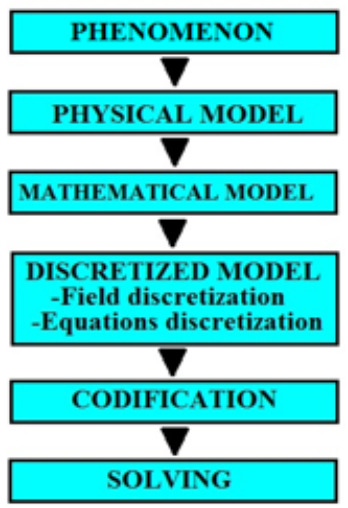

The differential equation of the dynamic equilibrium

The differential equation of the dynamic equilibrium describes the kinetic state of the system for the elemental volume:

$$
\frac{\partial \sigma_{i j}}{\partial x_{j}}+\rho f_{i}=\rho \frac{\partial^{2} U_{i}}{\partial t^{2}}
$$

The equation (1) will be derived to the limit:

-tension on border $\mathrm{S}_{\sigma}: \sigma_{y} n_{j}=p_{i}(t)$

-position on border $\mathrm{S}_{\mathrm{u}}: U_{i}=D_{i}(t)$

Legend for equations (1), (2) and (3):

$-U$ - position if observed point during process development;

$-\rho$-specific gravity;

- $f_{i}$-applied stress forces;

$-p_{i}$-pressure stress on the border;
- $D_{i}$ - resulted points of position changes;

$-n_{j}$ - cosine directors of the normal area.

\section{Geometric equations}

The geometric equations are expressed differentially describing the relation between deformations and displacements:

$$
\varepsilon_{i t}=\frac{1}{2}\left(\frac{\partial U_{i}}{\partial x_{i}}+\frac{\partial U_{i}}{\partial x_{i}}\right)
$$

In the event of large deformations, similar to those that occur at impact on the intended target, the approach is incremented. Following, the calculated distorted state made at the time $t$ becomes the initial condition for the next calculation interval, set up as the elementary time $d t$. To calculate the incremental geometric mean, the equations become:

$$
d \varepsilon_{u}=\frac{1}{2}\left(\frac{\partial\left(d U_{t}\right)}{\partial x_{t}}+\frac{\partial\left(d U_{t}\right)}{\partial x_{i}}\right)
$$

where $d$ represents base variations.

System deformation at any time is obtained by summing up the increases on calculation intervals:

$$
\varepsilon_{i j}=\int_{0}^{1} d \varepsilon_{i j}
$$

The deformations defined elementarily by relations (5) and (6), are called natural deformations.

\section{Physical equations}

Physical equations are constitutive equations, reflecting the physical link between stress, strain, speeds of displacement and temperature. The general form of physical equations is:

$$
F(\sigma, \varepsilon, \dot{\varepsilon}, T) .
$$

Analysis of the three sets of equations leads to the conclusion that the primary functions of the field in deformable solid mechanics - three shifts, six and six tensions deformations - are not independent.

Most of the methods used for describing the deformable solids limit the group of unknown factors to the three displacement factors - regarded as the primary unknown. After solving the displacements equations, the deformation values are obtained from the equation (5), and the values of tensions from the constitutive equations (7). The method of solving the system of displacement vectors of the equations governing the deformable solid mechanics is taken into the mathematical formalism calculation of the method using finite element criteria.

The discretization of the simulated model was done using a hexahedron net of 3D finite discrete spatial elements. The use of the hexahedron net enables the development of structured networks $(I, J, K)$, more flexible and easier to control than other types of dimensional networks. The network allows diversifying the system using finite element mesh on the field, as required. Thus, in regions where large gradients of primary functions are generated, the network can be refined.

A correct geometric discretization accurately generates acceptable solutions without excessive consumption of computing resources. The structured network with variable pitch is often used to build models with finite elements for the structural elements analyzed. Using this procedure, here were discretized key components such as: the impact stress loading including the characteristics of the incendiary piercing penetrator and ballistic protection package. Figure 
2 presents the impact configuration and details of the two components.

Empirical data derived for the materials used in the mathematical simulation

All types of material involved in the simulation models, from the simplest linear elastic to the most complex such as Johnson-Cook, are formulated by mathematical equations which include a range of material coefficients. The plastic Johnson - Cook model fully defines the flow tensor $\sigma$, taking into account deformation effects velocity and temperature in addition to the actual effect of plastic deformation. The plasticity ohnson - Cook is expressed in terms separately by the constitutive equation:

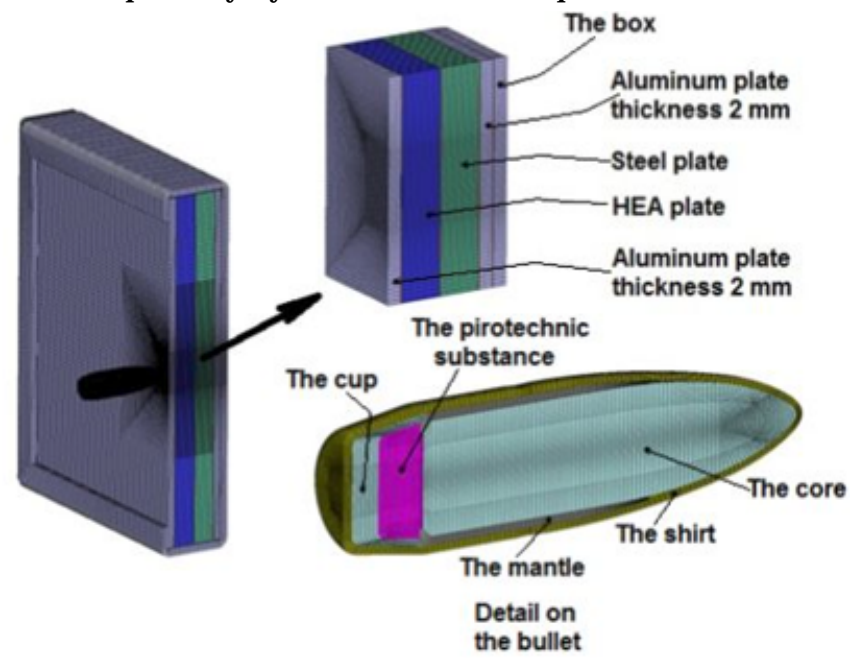

Fig.2. Virtual model of the impact between the projectlie and the BT.

$$
\sigma_{y}=\left(A+B \varepsilon_{p}^{*}\right)\left(1+C \ln \left(\frac{\dot{\varepsilon_{p}}}{\dot{\varepsilon}_{0}}\right)\right)\left(1-\left(\frac{T-T_{0}}{T_{s p}-T_{0}}\right)^{*}\right)
$$

where: $A+B \varepsilon_{p}^{n}$ - the exponential component already established;

$$
\begin{aligned}
& 1+C \ln \left(\frac{\varepsilon_{p}^{*}}{\varepsilon_{0}^{*}}\right) \text { - the speed of deformation factor; } \\
& 1-\left(\frac{T-T_{0}}{T_{\text {top }}-T_{0}}\right)^{m} \text { - the temperature factor; }
\end{aligned}
$$

$A, B, C$ - specific material constants;
$T_{,} T_{\text {too }}$ - the environment temperature and the specific melting to temperature;

$\varepsilon_{p}$ - the real plastic deformation;

$\dot{\varepsilon}_{p}^{p}$ - speed of plastic deformation;

$\dot{\varepsilon}^{p}$ - reference value defining temporal casualty as function of time unit used;

$T$ - local temperature in the BT at time t;

$n, m$ - numerical factors.

The mechanical coefficients of the materials used in this study were determined experimentally, involving a number of additional specific tests. For the J ohnson Cook models, the mechanical characteristics of the most important materials (core penetrator, plate steel, high entropy alloy - HEA) included in the physical model designed for the numerical simulation of the ballistic impact behavior of packages with penetrator piercing incendiary caliber $7.62 \mathrm{~mm}$, are shown in table 1 .

\section{Numerical simulation results}

Numerical simulation of kinetic impact processes between ballistic projectiles and BT uses, in most cases, finite element analyses methods. These methods use materials like Lagrange netw orks, i.e. a network attached to the bodies and which deforms together with them. In figure 3 can be seen in three successive images the penetration hole in the ballistic cassette components of the BT of the $7.62 \mathrm{~mm}$ caliber projectile. It should be noted that during the penetration process of the $\mathrm{BT}$, the projectile tip starts eroding and fragments from it are scattered in adjacent areas of the impact.

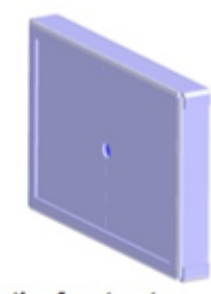

on the front entry

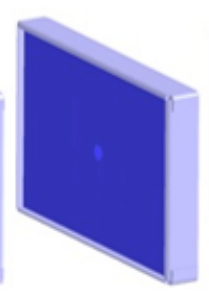

in the HEA plate

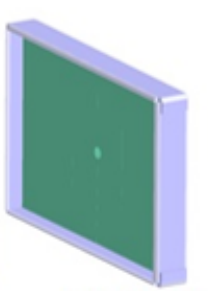

in the steel plate
Fig.3. Ballistic casette penetration structure after impact.

For a detailed analysis of the phenomenon and to draw comparisons with experimental validation of the simulation model, in figure 4 are shown the sequences of BT penetration process at different points in time.

From the figure, it can be noticed how the projectile (bullet) penetrates the steel and HEA plates forming the BT, but does not penetrate the ballistic frame, being retained by the last aluminum backing plate. Therefore, it can be said that all the projectile kinetic energy is

\begin{tabular}{|c|c|c|c|c|c|c|c|c|c|c|c|}
\hline \multirow{4}{*}{ 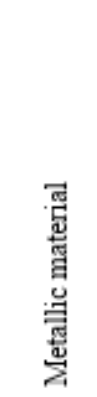 } & \multicolumn{11}{|c|}{ Mechanical characteristics } \\
\hline & \multirow[b]{2}{*}{$\begin{array}{l}\text { 窝 } \\
\text { 口̆ }\end{array}$} & \multirow[b]{2}{*}{ 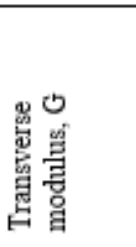 } & \multirow{2}{*}{ 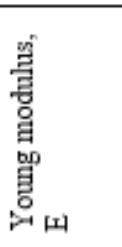 } & \multirow[b]{2}{*}{ 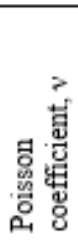 } & \multicolumn{7}{|c|}{ Johnson-Cook coefficients } \\
\hline & & & & & A & B & $\mathrm{n}$ & $\mathrm{C}$ & $\mathrm{m}$ & $T_{\text {top }}$ & $\mathrm{T}_{0}$ \\
\hline & $\mathrm{Kg} \mathrm{m}^{-3}$ & $\mathrm{MPa}$ & $\mathrm{MPa}$ & - & $\mathrm{MPa}$ & $\mathrm{MPa}$ & - & - & - & $\mathrm{K}$ & $\mathrm{K}$ \\
\hline HEA & 7720 & $0.8110^{5}$ & $2.210^{5}$ & 0.35 & 1550 & 1200 & 0.24 & 0.032 & 1.00 & 1850 & 300 \\
\hline $\begin{array}{l}\text { Armor } \\
\text { steel }\end{array}$ & 7850 & $0.8210^{5}$ & $2.110^{5}$ & 0.3 & 1250 & 3200 & 0.18 & 0.15 & 1.00 & 1763 & 300 \\
\hline
\end{tabular}

Table 1

CHARACTERISTICS OF MATERIALS AND THE JOHNSON - COOK COEFFICIENTS 


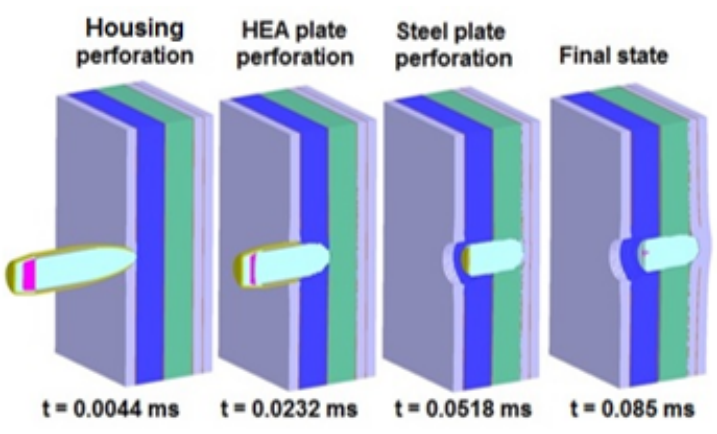

Fig.4. Sequential stages of the perforation process

consumed during perforating the steel and HEA plates, being held ultimately by the aluminum plates at the back of the frame.

Two important factors of the analyzed phenomenon are the projectile speed and its kinetic energy. The evolution with time of these is shown in figure 5 , which shows sequential axial velocity field of the $7.62 \mathrm{~mm}$ caliber projectile at impact time on the BT. Figure 5 also describes the projectile velocity reduction and its core erosion as it advances through the BT.

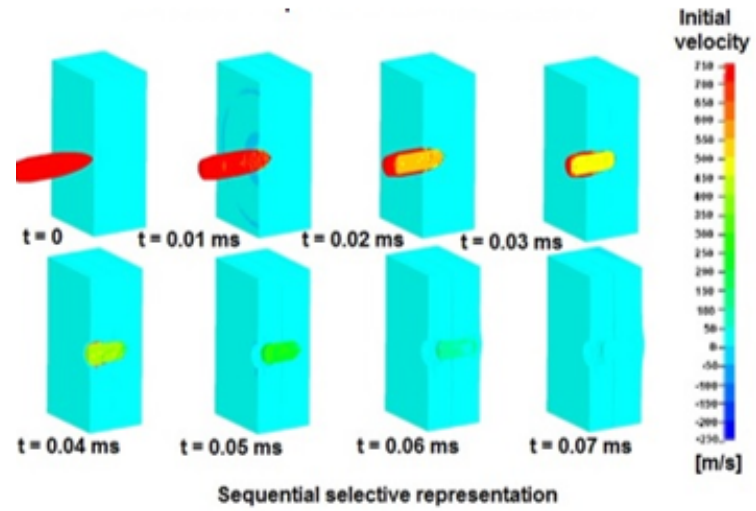

Fig.5. Projectile kinetic energy, speed and core variation during impact

The numerical simulation showed that during the impact of the piercing incendiary $7.62 \mathrm{~mm}$ projectile fired at $750 \mathrm{~ms}^{-1}$ speed and BT having as main components AlCrFeCoNi HEA and steel plates, the complete penetration of the target does nothappen. This was also demonstrated by determining the time variations of the speed and energy of the projectile core.

\section{Physical model of the ballistic multi-layer target}

To study the projectile-target interaction there was designed a physical model for the target, a sandwich composed of metallic, multi-wall sheets(aluminum, high entropy alloy and steel with good strength to dynamic stress). The design and construction of the experimental target is based on previous results of the mathematical analysis $[3,6]$, of the estimated data for the chosen materials, well defined by the current data base. To develop the physical model of the projectile impact with the multilayer target, several physical models were built, called Ballistic Target or BT, made of combinations of different layers of metallic materials. The BTs were used as targets for impact projectiles, to test their reaction and interaction with the projectile and kinetic fragments, with the direct objective of determining the level of protection that such system could offer to the manufacturing of individual and collective protective shields. The choice of the sandwich thickness, of the number and structural order of the layers in the composite BT was dictated by the efficiency of the shield protective property.
The materials chosen for production of the BT are part of the AlCrFeCoNi class, heat treated alloys [ $13-18,20$ 22] with high entropy and mechanical and physical properties adequate to such an application, steel with high resistance to dynamic stress and aluminum plates with the expressed role to reduce the freedom of movement inside the BT. The BT was packaged into a rectangular prism frame made of aluminum, to rigidize the two main metallic components (figs. 6 and 7) and contains a high entropy alloy plate with dimensions of $100 \times 100 \times 6 \mathrm{~mm}$, under which is positioned a steel plate, both plates sandwiched by $100 \times 100 \times 2 \mathrm{~mm}$ aluminum plates of the same general size. The structural cohesivity and reduced freedom of movement within the BT frame is enhanced by adhesive resin inside the sandwiched structure of a ballistic aluminum box frame. The edges of the box were pressed around the BT, leaving one side open to face the projectile during impact. The assembling procedure is illustrated in sequence in figure 5 and 6 .

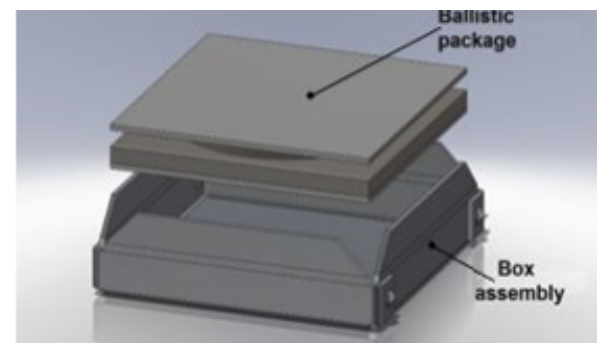

Fig.6. Positioning of BT inside the aluminum ballistic box

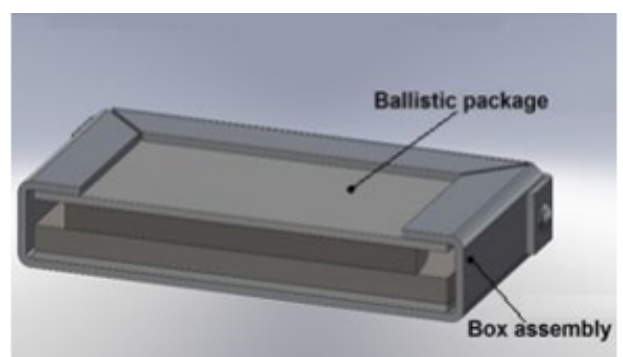

Fig.7. Section view of the BT
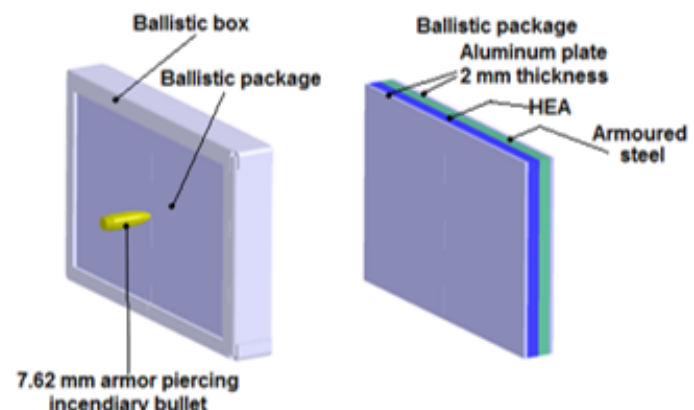

Fig.8. Physical model contained multi-layers used for numerical simulation: HEA+Steel (high entropy alloy + steel)

The data introduced in the mathematical model are the most accurate available data, describing the metallic and nonmetallic components material. The assembly target was impacted by a caliber of $7.62 \mathrm{~mm}$ penetrant incendiary bullet, with an assumed impact speed of $750 \mathrm{~ms}^{-1}$ of a military cartridge, to exceed the STANAG 4635, no. 2 minimum $695 \mathrm{~ms}^{-1}$, standard requirement speed for testing of shield protective performance.

The target model geometry was a result of transitory, non-linear phenomenon simulation using professional modeling software. Figure 8 shows the BT and projectile physical model at Time 0 exposing the position of the projectile vs. BT. 


\section{Results and discussions}

To validate the numerical model, a series of shooting range tests were performed using the experimental $\mathrm{BT}$, designed to determine the dynamic behavior of multi-layer structures made of steel, HEA and aluminum plates, in order to comply with the provision of operational requirements. Experimental tests were conducted on shooting range appropriate for such activities, using piercing-incendiary caliber $7.62 \mathrm{~mm}$ projectiles. The ballistic cassette (BT) was built in accordance with figures $6-8$, with final dimensions of $112 \times 108 \times 25 \mathrm{~mm}$, resulting from the use of impregnation resin. This was used to test the impact behavior against piercing incendiary projectiles of $7.62 \mathrm{~mm}$ caliber, and was mounted on a wooden stand, placed at $5 \mathrm{~m}$ distance from the firing weapon mouth (fig. 9).

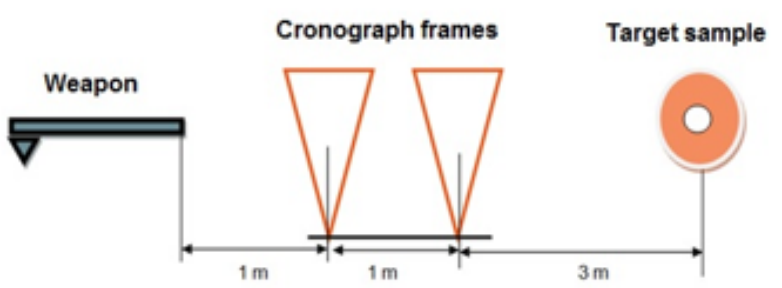

Fig.9. Experimental stand for dynamic test

\begin{tabular}{|c|c|c|c|c|}
\hline \multirow{2}{*}{ Experiments } & \multicolumn{4}{|c|}{ Test Parameters } \\
\cline { 2 - 5 } & Number of & Explosive load & $\begin{array}{c}\text { Projectile } \\
\text { travel }\end{array}$ & Impact effect \\
& shots & weight, g & speed, $\mathrm{ms}^{-1}$ & \\
\cline { 2 - 5 } & 6 & 1 & $415-427$ & BT unpenetrated \\
\hline Set 1 & 5 & 1.3 & $585-608$ & BT unpenetrated \\
\hline Set 2 & 15 & 1.6 & $710-727$ & BT unpenetrated \\
\hline Set 3 & & & & \\
\hline
\end{tabular}

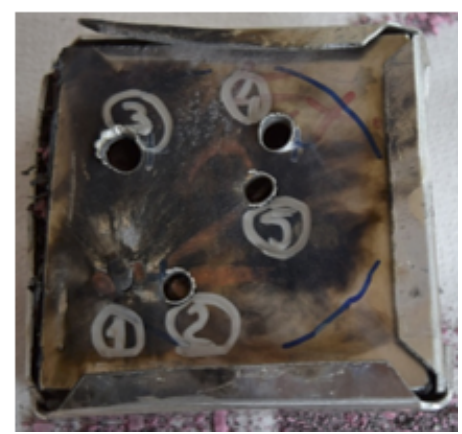

Fig. 10. BT front plate piercing

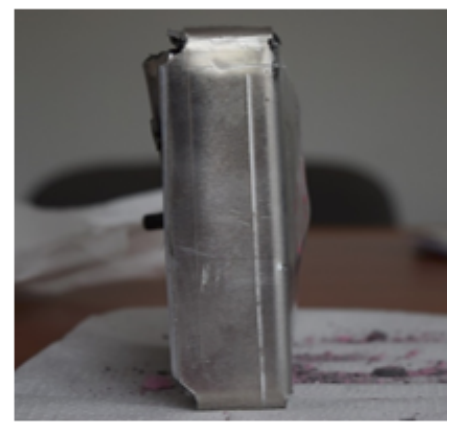

Fig.11. Side view of the ballistic frame
To determine the initial rate of the projectiles perpendicular to the direction of travel, a multiple-frame chronograph photocell was aligned. The test procedure involved varying the initial speed of the projectile to establish the speed at which all kinetic energy of the projectile is consumed for its plastic deformation and for the total or partial penetration of BT. Tests were performed at $25^{\circ} \mathrm{C}$ and a humidity of $55 \%$.

The characteristics of the ammunition used in the experimental tests were as follows:

-Ammunition type: piercing incendiary;

-Size: 7.62 x $39 \mathrm{~mm}$;

-Projectile weight: $7.67 \mathrm{~g}$;

-Core weight: $4 \mathrm{~g}$

To obtain the required initial velocity, the amount of powder in the cartridge was varied. The shooting program ( 26 shots) was made with different masses of explosive charge (table 2), as follows:

-Set 1 - explosive load weight of $1.0 \mathrm{~g}$;

-Set 2 - explosive load weight of $1.3 \mathrm{~g}$;

-Set 3-explosive load weight of $1.6 \mathrm{~g}$ (maximum speed).

Figures 10 and 11 show ballistic frames from the front and profile after experimental tests in the shooting range. It is to be noted that piercing-incendiary projectiles penetrated just the front of the BT, but failed to penetrate BT entirely. The experimental results perfectly validate the numerical simulation and modeling of the multi-layer system for individual and collective protection against high velocity projectiles. At the same time, these results can be considered in order to further develop other composite systems, such as: HEA -ceramic, HEA-Dyneema, HEAKevlar, HEA-Nylon etc., which are serious candidates for personal and collective armor applications.

\section{Conclusions}

Numerical simulation and modeling methods are excellent tools for examining quantitative mathematical processes and phenomena in the physics of materials. They can ensure maximum accuracy in the interpretation of dynamic evolution and the behavior of metallic materials or complex structures.

Modeling of composite structures, consisting of combinations of materials - aluminum alloy, high entropy alloy AICrFeCoNi and armor steel for military applications, was carried on real physical models, taking into account the mechanical characteristics of the materials and testing conditions. The ballistic package designed on the principle of multiple interfaces was composed of differentmaterials, having specific role, as follows: the front plate, made of high purity aluminum 99,5\%Al, absorbed a part of the impact energy of the projectile, by plastic deformation; the second plate made of high entropy alloy (AlCrFeCoNi) absorbed a large part of the impact energy, due to its toughness and high compressive strength; the third plate made of armor steel absorbed the final impact energy, by conversion into heat and plastic deformation; the backing plate of aluminum allowed the retention of fragments resulting from breakage of the previous plates. 
Experimental validation of the mathematical model was performed by a real impacttest, using a piercing-incendiary projectile with caliber of $7.62 \mathrm{~mm}$. The composite structures built and assembled into a ballistic frame, provided a ballistic protection againsta piercing-incendiary projectile of $7.62 \times 39 \mathrm{~mm}$ caliber, of up to $750 \mathrm{~ms}^{-1}$ speed. Compared to the target made of armor steel having the same wall thickness, the HEA experimental alloys used in the experiments show a higher aptitude to retain the projectile, under the same testing conditions.

For the future improvement of target design, the composite structure will be rebuilt to reduce the size of the metallic parts (HEA and armor steel) simultaneously with the addition of light alloy (Dural) or polymer.

Acknowledgment: The research work was financially supported by the Romanian National Program for Research in the framework of the Project No. PCCA 209/2012 Composite structures resistant to dynamic loadings applied at high deformation speeds used in the field of collective protection - HEAMIL" and by a grant of the Romanian Ministry of Research and Innovation, CCCDI - UEFISCDI, project number PNIII-P1-1.2-PCCDI-2017-0239/20 PCCDI 2018, Individual and collective protection systems for the military domain based on high entropy alloy - HEAPROTECT, within PNCDI III.

\section{References}

1.FREW, D.J., FORRESTAL, M.J ., CHEN, W. Pulse shaping techniques for testing elastic-plastic materials with a Split Hopkinson Pressure Bar. Experimental mechanics, 45, no. 2, 2005, p. 186-195.

2.GOVENDER, R.A., CLOETE, T.J., NURICK, G.N. A numerical investigation of dispersion in Hopkinson Pressure Bar experiments. J ournal de Physique IV, 134, 2006, p. 477-479.

3.ROTARIU, A., BUGARU, M., CHERECHES, T. Finite elements method in SHPB developing process. 6-th WSEAS International Conference on System Science and Simulation in Engineering, Venice, Italy, November 21-23, 2007.

4.FREW, D.J., FORRESTAL, M.J ., CHEN, W. Pulse shaping techniques for testing brittle materials with a Split Hopkinson Pressure Bar. Experimental mechanics, 42, no. 1, 2001, p. 93-106.

5 .JOHNSON, G.R. COOK, W.H. A constitutive model and data for metals subjected to large strains, high strain rates and high temperatures. Proc. 7-th International Symposium Ballistics, Netherlands, 1983, p. 541-547.

6.ROTARIU, A., CHERECHES, T. Dedicated software application for split Hopkinson pressure bar and its critical assessment. Annals of DAAAM for $2007 \&$ Proceedings of the 18-th International DAAAM Symposium, ISSN 1726-9679, ISBN 3-901509-58-5, Editor B. Katalinic, Published by DAAAM International, Vienna, Austria, 2007, p. 651- 652. 7.GORHAM, D.A. A numerical method for the correction of dispersion in pressure bar signals. Journal of Physics E: Scientific Instruments, Vol. 16, no. 6, 1983, ISSN 002-3735, p. 477-479.

8.HOUSE, J.W., LEWIS, J.C., GILLIS, P.P., WILSON, LL. Estimation of flow stress under high rate plastic deformation. International J ournal Impact Energy, no. 2, 1995, p. 189-200.

9.ERLICH, D.C., CHARTAGNAC, P. Determination of dynamic flow curve of metals at ambient and elevated temperatures by rod impact techniques. J. Phys. France 46, 1985, p. 455-462.

10.DONG, Y., FENG, S., HUANG, G., LIU, C., XIAO, L., SONG, Q. Ballistic impact characteristics of flat-nose projectile penetrating concrete and soil compound target. International Journal of Nonlinear Sciences and Numerical Simulation, 13, 3-4 , 2012.
11. HUANRAN WANG, CANYUAN CAI, DANIAN CHEN, DONGFANG MA. Dynamic Constitutive Behavior and Fracture of Lanthanum Metal Subjected to Impact Compression at Different Temperatures and Impact Tension. International Journal of Nonlinear Sciences and Numerical Simulation, 14, 1, 2013.

12.WU, H-J., HUANG, F-L., WANG, Y-N., DUAN, Z-P., SHAN, Y. Mass Loss and Nose Shape Change on Ogive-nose Steel Projectiles During Concrete Penetration. International J ournal of Nonlinear Sciences and Numerical Simulation, 13, 3, 2012.

13.WANG, Y.P., LI, B.S., REN, M.X., YANG, C., FU, H.Z. Microstructure and compressive properties of $\mathrm{AlCrFeCoNi}$ high entropy alloy. Materials Science and Engineering A, 491, 2008, p. 495-500.

14.GEANTA, V., VOICULESCU, I., STEFANOIU, R., SAVASTRU, D., CSAKI, I., PATROI, D., LEONAT, L. Processing and characterization of advanced multi-element high entropy materials fron AICrFeCoNi system. Optoelectronics and Advanced Materials -Rapid Communications, 7, 11-12, 2013, p.874-880.

15. VOICULESCU, I., GEANTA, V., STEFANOIU, R., PATROI, D., BINCHICIU, H. Influence of the Chemical Composition on the Microstructure and Microhardness of AlCrFeCoNi High Entropy Alloy. Rev. Chim. (Bucharest), 64, no. 12, 2013, p.1441-1444.

16.KAOA, Y-F., CHENA, T-J., CHENB, S-K., YEH, J-W. Microstructure and mechanical property of as-cast, homogenized, and deformed AlxCoCrFeNi $(0 \leq x \leq 2)$ high-entropy alloys. Journal of Alloys and Compounds, xxx $2009 x x x-x x x$, p.1-8.

17.SHUN, T-T., DU, Y-C. Microstructure and tensile behaviors of FCC Al0.3CoCrFeNi high entropy alloy. Journal of Alloys and Compounds, 479, 2009, p.157-160.

18.STEFANOIU, R., GEANTA, V., VOICULESCU, I., CSAKI, I., GHIBAN, N. Researches regarding the influence of chemical composition on the properties of AlxCrFeCoNi alloys. Rev. Chim. (Bucharest), 65, no. 7, 2014, p.819-821.

19*** www.uefiscdi.ro. Structuri compozite rezistente la solicitari dinamice cu viteze mari de deformare cu aplicabilitate in domeniul protectiei colective -Composite structures resistant at dynamic loadings applied with high deformation speeds used in the field of collective protection - HEAMIL -PCCA 209/2012.

20.GEANTA, V., VOICULESCU, I., MILOSAN., ISTRATE, B., MATES, I.M. Chemical Composition Influence on Microhardness, Microstructure and Phases Morphology of $\mathrm{Al}$ CrFeCoNi High Entropy Alloys, Rev. Chim. (Bucharest), 69, no.4, 2018, p.798-801.

21.NAHMANY, M., HOOPER, Z., STERN, A., GEANTA, V., VOICULESCU I. AlxCrFeCoNi High-Entropy Alloys: Surface Modification by Electron Beam Bead-on-Plate Melting, Metallography Microstructure and Analysis, 5, 3, 2016, p.229-240.

22.CSAKI, I., STEFANOIU, R., GEANTA, V., VOICULESCU, I., SOHACIU, M.G., SOARE, A., POPESCU, G., SERGHIUTA, S. Researches regarding the processing technique impact on the chemical composition, microstructure and hardness of AlCrFeNiCo high entropy alloy, Rev. Chim. (Bucharest), 67, no. 7, 2016, p. 1373-1377.

23.SPANU A., BESNEA, D., VOICULESCU, I. Improvement of Polymer Surface Properties Using the Deposition with Thin Metal Alloy Layers, Mat. Plast., 55, no. 2, 2018, p.141-145,

24.BUZATU, M., GHICA, S.I., PETRESCU, M.I., GEANTA, V., STEFANOIU, R., IACOB, G., BUTU, M., VASILE, E. Obtaining and characterization of the Ti15M05W alloy for biomedical applications. Mat. Plast. 54, no.3, 2017, p.596-600.

\section{Manuscript received: 14.01 .2019}

